\title{
Documentation of Pregnancy Status Before Surgery in Kenya
}

\author{
Alex Muturi, ${ }^{1}$ Mohamed Omar, ${ }^{2}$ Brian Ngure, ${ }^{3}$ Daniel Ojuka, ${ }^{3}$ John Kinuthia ${ }^{4}$ \\ 1 Tigoni District Hospital, Kiambu \\ ${ }^{2}$ Mbagathi District Hospital, Nairobi \\ ${ }^{3}$ University of Nairobi \\ ${ }^{4}$ Kenyatta National Hospital
}

Correspondence to: Dr. Alex Muturi, PO Box 14523, Nairobi; email: almuturi@gmail.com

\begin{abstract}
Background: Performing surgical procedure on a pregnant woman may have major consequences for the fetus, patient, healthcare worker and institution. Assessment of pregnancy status in women of reproductive age when admitted to hospital is therefore an important safety practice. Documentation of likelihood of pregnancy among women admitted in the surgical units of Kenyatta National Hospital $(\mathrm{KNH})$ is not known. Objective: To assess documentation of pregnancy status and possibility of pregnancy before surgery at $\mathrm{KNH}$. Methods: This was a retrospective study involving all surgical units of female patients of reproductive age with various diagnoses and scheduled to undergo surgery between January 2011 and December 2016. Data collected included documentation of age, parity, last menstrual period, level of education, use of family planning, and pregnancy status confirmation using urine or serum beta human chorionic gonadotrophin (HCG) and ultrasound. Results: We analysed data from
\end{abstract}

331 patient records. All (100\%) of the sampled records had age of the patient recorded, $43 \%$ had information on parity documented, $35 \%$ had last normal menstrual period recorded, and only $26 \%$ of the records showed information on use of family planning. 19 (5.7\%) patients were confirmed to be pregnant using ultrasound and urine $\beta$-HCG. Conclusion: Although only a small proportion of women admitted in surgical units were pregnant, data on likelihood of pregnancy as deduced from information on age, last menstrual period and use of family planning were missing.

Keywords: Pregnant, Fetus, Safety, Surgery, Anaesthesia Ann Afr Surg. 2020; 17(1):7-10.

DOI: http://dx.doi.org/10.4314/aas.v17i1.3

\section{Conflicts of Interest: None}

Funding: None

(C) 2020 Author. This work is licensed under the Creative Commons Attribution 4.0 International License.

\section{Introduction}

In up to $2 \%$ of all pregnancies, general anaesthesia is needed in non-obstetrical surgical cases (1). Early pregnancy complications such as ectopic pregnancy, premature labor, chorioamnionitis, and abruption placentae may present as acute abdomen and present to the surgical units (2). In addition, surgical pathologies such as appendicitis, cholecystitis, intestinal obstruction and trauma may pose risks to the fetal, placental and maternalwellbeing through such mechanisms as maternal hypoxia, acidosis and alterations in uteroplacental blood flow (2-4). Currently, no anaesthetic agent has been shown to have direct teratogenic effects on the human fetus $(1,4)$. However, various studies report that all inhalation anaesthetic agents have teratogenic effect on certain species at various points during their gestational periods under certain conditions $(1,4,5)$. In the perioperative setting, pregnant women may be exposed to ionizing radiation from radiological evaluation and this is potentially teratogenic to the fetus, especially in the first trimester $(4,5)$. A large number of pregnancies especially in the first trimester are unrecognized by both the physician and the patient (5). The current practice is to postpone elective surgery in patients during pregnancy due to the risks the fetus is exposed to $(1,4,5)$. The cost of identifying a pregnancy may be high as the incidence rates are low; however, the associated damage that is present in case of a miscarriage or a child who is born with a congenital anomaly is irreparable and may lead to medicolegal, psychological and psychosocial costs that could have been prevented using a simple preoperative test (5). The National Institute for Health and Care Excellence (NICE, USA) guidelines indicate that pregnancy status should be documented before undertaking any elective surgical procedure in ladies of reproductive age (6). The National Patient Safety Agency (NPSA) in 2010 published a report in which they advocate preoperative assessment of pregnancy status in 
females of reproductive age group and integrating this assessment as part of the preoperative documentation used by staff performing the final clinical and identity checks before initiating surgery (7). The American Society of Anesthesiologists recommends stratifying patients and adds that pregnancy testing may be offered to female patients of childbearing age for whom the result would alter the patient's medical management (8). Identifying a pregnancy preoperatively in a female of childbearing age minimizes risks to the mother and the fetus and also the attendant medical-legal challenges (4,9-11). Currently, no data exist in Kenya that document the incidence of pregnancy in females of childbearing age who are to undergo elective or emergency non-obstetric surgical procedures. Kenyatta National Hospital's (KNH) preoperative checklist does not define determination of pregnancy status in females of childbearing age who are to undergo surgical procedures. This study sought to assess documentation of pregnancy status and the possibility of pregnancy before surgery at the hospital.

\section{Methodology}

This was a retrospective study involving all surgical units, of female patients of reproductive age with various surgi-cal diagnoses who were scheduled to undergo emergency and elective surgery from January 2011 to December 2016. KNH has a capacity of 1,600 beds, and 24 outpatient and specialist clinics. Ethical approval was sought for and granted (ERC P415/07/2017). For the purpose of this study, reproductive age ranged from 12 to 55 years: most recorded births in our context are within this range. We also used the age cut because we wanted to study females admitted in adult wards, which in our set up is from 12 years. Fifty-five-year limit was chosen as the most commonly referenced median age for menopause. We excluded ladies admitted for nonsurgical reasons. Data collected from patients' records included documentation by clinician at admission of age, parity, last menstrual period, use of family planning, and pregnancy status confirmation (using urine $\beta$-HCG test, ultrasound, serum $\beta$-HCG). We analyzed data for proportion of patients documented and looked at significant factors that determined documentation. Statistical significance was p-value $<0.05$.

\section{Results}

Of the expected 383 files, we analysed 331 records because the rest were missing or were wrongly labelled. Of the analysed files, $100 \%$ had age documented, 329
(99.4\%) had marital status documented, 125 (37.8\%) did not have level of education documented at all. Obstetric data documented included parity for 142 (42.9\%) and last normal menstrual period for 117 (35.3\%) files (Table 1). The level of education was documented for $206(62.2 \%)$ patients with most having secondary school level, none $2.1 \%$, primary $20.8 \%$, secondary $26.3 \%$ and tertiary $13 \%$.

Table 1. Obstetric data

\begin{tabular}{lc}
\hline Variable & Frequency $(\%)$ \\
\hline Parity & $142(42.9)$ \\
Yes & $189(57.1)$ \\
No & \\
Gravidity & $17(5.1)$ \\
Yes & $314(94.9)$ \\
No & \\
Last menstrual period & $117(35.3)$ \\
Yes & $214(64.7)$ \\
No & \\
Family planning method & $85(25.7)$ \\
Yes & $246(74.3)$ \\
No & $19(5.7)$ \\
Documentation of pregnancy via ultrasound or $\beta-H C G$ \\
Yes & $312(94.3)$ \\
No & $330(99.7)$ \\
Abdominal exam findings, Fundal height finding \\
Yes & $5(1.5)$ \\
No & $326(98.5)$ \\
Heart sounds & \\
Yes & $526(98.5)$ \\
No & \\
Yoss to pregnancy & \\
No & \\
\hline
\end{tabular}

\section{Discussion}

Recognition of pregnancy status is possible only through a thorough reproductive history, physical examination and imaging or laboratory confirmation (11-13). Offering safe and quality care to these women is a challenge without complete documentation of the biodata, reproductive history, physical examination, and laboratory, urine or blood tests $(11,13,14)$. Level of education is likely to influence awareness of menstrual cycle, uptake of contraception and bargaining for safer sex.

It is important to capture education level as it adds to information that determines overall likelihood of pregnancy and therefore helps to select those who 
will consent to and be offered testing (15). Level of education is inversely related to risk of unrecognized and, often, unwanted pregnancy $(15,16)$.

Documentation of reproductive history was suboptimal with parity being captured in only $57 \%$ of the records. Last menstrual period and use of family planning were documented in two-thirds and three-quarters of the records respectively. Though there are limitations on the use of menstrual dates to determine likelihood of pregnancy, this data is vital in assessing likelihood of a woman of childbearing age being pregnant in order to select those that can then be tested $(17,18)$. The exception would be in cases of trauma where all women of childbearing age are tested for pregnancy as a precautionary measure $(2,19)$. As it is difficult to ascertain cost effectiveness of testing all women, it is important to document this reproductive history as an important patient safety measure $(2,20)$.

Abdominal examination may have been done, depending on the surgical diagnosis, and in the five patients confirmed to be pregnant the fundal height was recorded where it was palpated. For pregnancy greater than 12 weeks, a thorough abdominal examination would demonstrate an enlarged uterus and its documentation helps in planning to confirm the pregnancy and special attention is taken during the rest of the care to safeguard the wellbeing of the woman and the fetus $(11,13,21)$. This study reports a pregnancy rate of $5.7 \%$ among women of childbearing age admitted in various surgical units at $\mathrm{KNH}$. This rate is much higher than the quoted global average of $1-2 \%$. We also report a higher rate of documentation of vital information that relates to likelihood of pregnancy than in other previous work done in the UK $(1,2,9,22)$. While this higher rate could be due to the size of the sample considered, it is worth paying attention to the finding in policy making as far as safety of this category of patients is considered. Measures should be instituted to capture the reproductive history as part of admission data that will then be used to guide who gets tested.

Thiswas a retrospective study, with lack of documentation and some records possibly misplaced or lost. We did not relate documentation with outcomes. While this would no doubt have yielded a lot more data that would inform practice, it would have taken longer and cost more as the authors had time and financial constraints.

\section{Conclusion}

The documentation of vital information about reproductive status and likelihood of pregnancy among women of childbearing age admitted in surgical units at $\mathrm{KNH}$ is incomplete. This inadequacy may have an adverse effect on the safety of these women and their babies should they get anaesthesia and undergo surgery without the necessary precautions.

\section{References}

1. Maher JL, Mahabir RC. Preoperative pregnancy testing. Can J Plast Surg. 2012; 20(3):e32-4.

2. Augustin G, Majerovic M. Non-obstetrical acute abdomen during pregnancy. Eur J Obstet Gynecol Reprod Biol. 2007; 131(1):4-12.

3. Coleman MT, Trianfo VA, Rund DA. Non-obstetric emergencies in pregnancy: Trauma and surgical conditions. Am J Obstet Gynecol. 1997; 177(3):497-502.

4. Duncan PG, Pope WD, Cohen MM, et al. Fetal risk of anesthesia and surgery during pregnancy. Anesthesiology. 1986; 64(6):790-4.

5. Shnider S, Webster G. Maternal and fetal hazards of surgery during pregnancy. Am J Obstet Gynecol. 1965; 92(7):891900.

6. O'Neil F, Carter E, Pink N, et al. Routine preoperative tests for elective surgery: Summary of updated NICE guidance. BMJ. 2016; 354:i3292.

7. Lamont T, Coates T, Mathew $\mathrm{D}$, et al. Checking for pregnancy before surgery: Summary of a safety report from the National Patient Safety Agency. BMJ. 2010; 341:c3402.

8. Barbeito A, Muir H, Gan T, et al. Use of a modifier reduces inconsistency in the American Society of Anesthesiologists Physical Status Classification in Parturients. Anesth Analg. 2006; 102(4):1231-3.

9. Juhasz-Böss I, Solomayer E, Strik M, et al. Abdominal surgery in pregnancy-An interdisciplinary challenge. Dtsch Arztebl Int. 2014; 111(27-28):465-72.

10. García N, Morant J, González E. Non-obstetric surgery during pregnancy. Rev Colomb Anestesiol. Bogotá. 2011; 39(3):360-73.

11. Twersky R, Singleton G. Preoperative pregnancy testing: "Justice and testing for all." Anesth Analg. 1996; 83(2):4389.

12. Kuczkowski K. Nonobstetric surgery during pregnancy: What are the risks of anesthesia? Obstet Gynecol Surv. 2004; 59(1):52-6.

13. Speroff L, Fritz M. Clinical gynecologic endocrinology and infertility. 7th ed. Philadelphia, PA: Lippincott Williams \& Wilkins; 2005.

14. Paul M, Schaff E, Nichols M. The roles of clinical assessment, human chorionic gonadotropin assays, and ultrasonography in medical abortion practice. Am J Obstet Gynecol. 2000; 183(2 Suppl): S34-43.

15. Calvert C, Baisley K, Doyle A, et al. Risk factors for unplanned pregnancy among young women in Tanzania. J Fam Plann Reprod Health Care. 2013; 39(4):e2.

16. Koch E, Calhoun B, Aracena P, et al. Women's education level, contraceptive use and maternal mortality estimates. Public Health. 2014; 128(4):384-7.

17. Cole L, Ladner D, Byrn F. The normal variabilities of the menstrual cycle. Fertil Steril. 2009; 91:522-7.

18. Wilcox A, Baird D, Dunson D, et al. Natural limits of pregnancy testing in relation to the expected menstrual period. JAMA. 2001; 286(14):1759-61. 
19. G Jain, Chari R, Maslovitz S, et al. Guidelines for the management of a pregnant trauma patient. J Obstet Gynaecol Can. 2015; 37(6):553-7.

20. Manley S, de Kelaita G, Joseph N, et al. Preoperative pregnancy testing in ambulatory surgery: Incidence and impact of positive results. Anesthesiology. 1995; 83(4):6903.

21. Valenzuela R, Iserson K, Punguyivre D. False-positive urine pregnancy tests-Clinicians as detectives. Pan Afr Med J. $2011 ; 8: 41$.
22. Powell-Bowns M, Wilson M, Mustafa A. Documentation of pregnancy status, gynaecological history, date of last menstrual period and contraception use in emergency surgical admissions. Time for a change of practice? World J Surg. 2015; 39(12):2849-53. 